\title{
Unnötige Forderungen von santésuisse und Helsana
}

argomed - die Aargauer Hausärzte
Santésuisse verlangt am 9. Januar 2006 in einem Positionspapier mehr Qualitätskontrollen. Neben den ebenfalls kritisierten Spitälern würden bei den freipraktizierenden Ärzten Konzepte und Programme zur Qualitätssicherung fehlen. Helsana doppelt an ihrer Jahresmedienkonferenz nach und fordert, die medizinische Versorgung endlich durch Qualitätskontrollen transparenter zu machen.

Die argomed Ärzte AG vertritt 288 Grundversorgerinnen und Grundversorger (Allgemeinärzte, Allgemeininternisten und Kinderärzte), die in regionalen Ärztenetzen im Kanton Aargau organisiert sind. Wir fühlen uns angesprochen, zu den Forderungen Stellung zu nehmen und Richtigstellungen anzubringen.

Egal ob diese Vorwürfe aus Ignoranz oder aus bösem Willen erhoben werden, sie treffen nicht zu! Qualitätskontrollen und Qualitätsverbesserungen wurden in der Medizin schon immer und werden auch heute laufend umgesetzt, nicht erst, seit es das neue KVG gibt. Es sind aber nicht primär die Krankenversicherer, sondern die staatlichen Stellen, welche die Qualität überwachen und für die Sicherheit der Patienten in Arztpraxen und Spitälern verantwortlich sind. Zur Erinnerung der Versicherer zählen wir die Gesetze, Verordnungen, Reglemente und die anderen Vorschriften auf, welche in Arztpraxen befolgt werden müssen. Spitäler unterstehen noch vielen weiteren Vorschriften, auf deren Aufzählung wir an dieser Stelle verzichten.

\section{Strukturqualität}

Die Zulassung zum Studium und das Studium selbst sind staatlich geregelt und werden geprüft (Staatsexamen).

Die Weiterbildung zum Facharzt ist in ausführlichen Reglementen geregelt und untersteht der Kontrolle des BAG.

Die MPA-Ausbildung untersteht dem Bundesamt für Berufsbildung. Ähnliche Regelungen bestehen für Arztsekretärinnen, Pflegefachpersonen usw.

Die laufende Fortbildung der Fachärzte ist in Reglementen festgelegt, wird von den Fachverbänden überprüft und vom BAG beaufsichtigt.
Auch das Praxispersonal bildet sich laufend fort.

\section{Röntgen}

Röntgengerät und Röntgenraum werden vom Strahlenschutz abgenommen. Jährliche Wartungen und Überprüfungen durch Röntgenfirmen gehören ebenso wie die wöchentlichen Konstanzprüfungen $\mathrm{zu}$ den klaren Pflichten und werden vom BAG beaufsichtigt. Das eingesetzte Personal muss die vorgeschriebene Ausbildung nachweisen. Die Ärzte, welche die Verantwortung tragen, mussten sich in den vergangenen Jahren aufwendigen Strahlenschutzausbildungen und -prüfungen unterziehen, ohne dass die Ärzte hierfür eine höhere Abgeltung gefordert hätten.

\section{Labor}

Auch hier bestehen regelmässige, kostspielige externe und interne Qualitätskontrollen, beispielsweise Ringversuche, die von den MPA durchgeführt werden. Mit der Senkung des Labortaxpunktes wird dieser Mehraufwand, der die hohe Qualität der Untersuchungen garantiert, zu einer immer höheren Belastung.

\section{Sterilisation}

Die Anforderungen an die Apparate sind aufgrund von Verordnungen erhöht worden.

Bis hin zur Messung der Kühlschranktemperatur, die täglich monitorisiert werden muss, unternimmt die Ärzteschaft vieles zur Sicherung der Strukturqualität.

Leider ist der Nutzen einzelner Massnahmen bereits in einem Grenzbereich angelangt, in welchem die dadurch erzielte Qualitätsverbesserung nicht mehr ersichtlich ist und für die Patientensicherheit irrelevant wird. Qualitätskontrolle darf nicht mit übertriebener Bürokratie gleichgesetzt werden. Übertriebene Anforderungen an die Strukturqualität gefährden bei beschränkten Ressourcen die Prozess- und die Outcome-Qualität.

Weitere Strukturqualitäten erfüllen Ärztenetze mit regelmässigen netzinternen Veranstaltungen zur Weiterbildung der Netzärzte und des 
Personals, definierten Beitritts- und Mitgliedschaftskriterien, elektronischer Vernetzung zum Daten- und Informationsaustausch sowie Anlauf- und Ombudsstellen für Patienten.

\section{Prozessqualität}

Alle Fachärzte müssen jedes Jahr eine hohe Zahl von Fortbildungsstunden nachweisen, in Form von Fortbildungsveranstaltungen, Kongressen, elektronischen Lernprogrammen (CME Current Medical Education) und Studium von Fachliteratur. Über die Erfüllung der Fortbildungsrichtlinien wachen die entsprechenden Fachgesellschaften. Regionale Fortbildungsveranstaltungen dienen der Vernetzung zwischen Grundversorgern, Spezialärzten und Spitalärzten und leisten unschätzbare Dienste bei der reibungslosen Betreuung unserer Patienten.

Darüber hinaus sind die Netzärzte in den Ärztenetzen zur aktiven und regelmässigen Teilnahme an Qualitätszirkeln verpflichtet. Das Überweisungsverhalten, die Medikamentenverschreibung und Behandlungsrichtlinien (Guidelines) werden diskutiert und an die eigenen Bedürfnisse angepasst. Die Zusammenarbeit innerhalb des Ärztenetzes und mit netzexternen Leistungserbringern wird ständig verbessert und weiterentwickelt. Neue Ärzte werden eingeführt und begleitet. Ebenso findet ein überregionaler Austausch der Ärztenetze statt. Weiter werden Instrumente zur Qualitätsverbesserung umgesetzt, die von der Dachorganisation der Hausärzte vorangetrieben werden und an deren Implementierung die Hausarztnetze derzeit arbeiten (z.B. Qualitätsindikatoren Quali-mednet). Die gezielte Datenauswertung und -analyse ist für Ärztenetze heute selbstverständlich.

argomed hat in Anlehnung an die SGAM ein CIRS Critical Incident Monitoring System in Betrieb genommen. Analog zur Fliegerei und zur Anästhesie werden Fehlermeldungen gesammelt, um auf kritische Abläufe aufmerksam zu machen und zu ihrer Reduktion beizutragen.

Eine eben ausgewertete Umfrage unter Grundversorgern hat ergeben, dass gegen 20\% von ihnen trotz höherem Arbeitsaufwand bereits regelmässig elektronische Hilfsmittel bei der Verschreibung von Medikamenten verwenden, die die Sicherheit der Pharmakotherapie durch Interaktionskontrolle und Vermeidung von Lesefehlern erhöhen. Die Zusammenarbeit der Hausärzte mit den Versandapotheken zur Rose und Mediservice hat die Einführung dieser Instrumente erst erlaubt. Bei der Einführung solcher Qualitätsmassnahmen wäre eine Unterstützung durch die Krankenversicherer und/oder die Apotheker sehr erwünscht.

\section{Outcome-Qualität}

Bekanntlich ist die Outcome-Qualität sehr schwierig nachzuweisen. Die anhaltend steigende Lebenserwartung trotz ungesunder Lebensführung ist ein Indiz für den steigenden Nutzen der Medizin. Die Patienten berücksichtigen sehr wohl qualitative Aspekte bei der Wahl der Ärzte. Zunehmend werden durch die Grundversorger auch Forschungsprojekte/-studien durchgeführt und deren Resultate publiziert.

In den Ärztenetzen werden heute bereits verschiedene Instrumente eingesetzt:

- Patientenbefragungen und Praxisevaluationen (z. B. EPA) sind sinnvolle Instrumente, die angewendet werden können und dem Arzt einen Lerneffekt bringen;

- netzinterne Umfragen und Erhebungen;

- Disease- und Case-Management;

- Effizienz- und Wirtschaftlichkeitsstudien.

Die Helsana interpretiert die steigende Versichertenzahl als Indiz für die Zufriedenheit mit ihren Leistungen, konsequenterweise müsste sie die zunehmende Beanspruchung medizinischer Leistungen ebenfalls als Qualitätsbeweis für die Medizin anerkennen.

\section{Bemerkungen und Kritik}

Grundsätzlich müssen wir festhalten, dass für uns Ärztinnen und Ärzte die fachliche Qualität im Vordergrund steht. Wir lehnen Qualitätsmassnahmen ab, die allein auf eine Zertifizierung abzielen. Zuständig für die Umsetzung der Qualitätsvorschriften des KVG sind die Bundesbehörden und nicht die Krankenversicherer.

Wir sind überzeugt, mit diesen Ausführungen zu einem sachlicheren Umgang mit Qualitätsforderungen beizutragen. Forderungen aufzustellen ist einfach. Konstruktive Beiträge vermissen wir hingegen in den Stellungnahmen der Versicherer. Wir verstehen darunter auch die Bereitschaft für eine adäquate Entschädigung des Mehraufwands für die geforderten Qualitätsmassnahmen.

Insbesondere möchten wir unser Bedauern ausdrücken, dass trotz mehrfacher Einladung kein Vertreter von santésuisse am Symposium des med-swiss.net am 22. September 2005 teilgenommen hat, an welchem die grundlegenden Qualitätsindikatoren für Ärztenetzwerke (Qualimed-net ${ }^{\circledR}$ ) vorgestellt wurden, welche von den Ärztenetzwerken entwickelt wurden. Diese Geringschätzung trifft uns um so mehr, als nachher in ihrem Positionspapier der Vorwurf erhoben wird, dass sich die Ärzte nicht um die Qualität ihrer Arbeit bemühen. 